\title{
ANÁLISE E AVALIAÇÃO DA TEORIA DE GERENCIAMENTO DE FILAS COM COLETA DE TEMPO EM UM LABORATÓRIO HOSPITALAR
}

\author{
Ana Carolina Nogueira Lima* - FIC/UNIS - anacarolinanogueiralma86@ gmail.com.br \\ Jaqueline Bispo Carvalho*- FIC/UNIS - jaquelineb_85@hotmail.com \\ Juliana Evangelista Costa* - FIC/UNIS - julianaevangellista@ hotmail.com \\ Juliana Silva Medeiros* - FIC/UNIS - julianasrmedeiros@gmail.com.br \\ Vinicius de Assis Oliveira*- FIC/UNIS - viniciusoliveira375@yahoo.com.br
}

\begin{abstract}
RESUMO: O presente trabalho foi realizado com objetivo de analisar como funciona o sistema de atendimento do laboratório Labclin no Hospital de Cataguases-MG, com ferramentas da disciplina de Pesquisa Operacional. A pesquisa se baseia em avaliar o método do gerenciamento de fila do sistema FIFO e implantar o SJF - NP com o objetivo de melhorar o processo. Os resultados mostraram que, no dia 23 de novembro de 2018, durante 3 horas de atendimento, a diferença do tempo de atendimento do FIFO para o SJF-NP reduziu em cerca de 39 minutos.

Palavras chaves: FIFO, gerenciamento de filas, redução no tempo de espera
\end{abstract}

\section{INTRODUÇÃO}

Para o bom funcionamento de um hospital é necessário atender a uma série de normas, ter profissionais capacitados, bom atendimento, eficiência, além de sistema de apoio ao diagnóstico. Quando pacientes procuram tratamentos médicos, esses quase sempre solicitam exames para quantificar ou constatar de fato uma enfermidade. Esses exames médicos aumentam as chances de cura, pois há um diagnóstico com precisão e rapidez, melhorando o processo de cura. "O aumento das filas nas emergências dos hospitais está relacionado à superlotação dos leitos, de acordo com Roberto José Bittencourt, médico e doutorando da ENSP. Em artigo na revista Cadernos de Saúde Pública (Vol. 25. No7)".

Todavia, se os processos de atendimentos gerarem filas demasiadamente grandes poderiam atrasar consultas, atendimentos marcados para o mesmo dia e até mesmo dificultarem atendimentos emergenciais. Além disso, surto de doenças epidemiológicas (dengue, febre amarela, entre outras) aumentam as filas para atendimento.

Este artigo buscou coletar o tempo em que um paciente leva para ser atendido na recepção de um laboratório de análises clínicas, o Labclin, situado na cidade de Cataguases, Minas Gerais.

\section{METODOLOGIA}

Para realização do presente trabalho foi realizada uma pesquisa bibliográfica em livros e artigos acadêmicos sobre a prática da disciplina pesquisa operacional. Os membros da equipe de trabalho em reunião e através de brainstorm decidiram realizar o trabalho no Laboratório de Análises Clínicas (Labclin) que funciona no laboratório do Hospital de Cataguases.

A visita e coleta de dados aconteceram no período de 19/11/18 a 23/11/18 a mesma foi realizada no período da parte da manhã, de acordo com a disponibilidade de horários dos pesquisadores. O horário de atendimento do Labclin é $24 \mathrm{hs}$, porém, não realiza atendimentos ao público na parte da noite, exceto em casos de urgência e emergência e apenas internos do hospital que, neste caso, não é o foco do trabalho, por não gerar fila de atendimento.

Para a coleta de dados, o pesquisador observou um grande número de pessoas na fila aguardando, medindo o tempo gasto para o atendimento e levando em consideração o tipo de exame. Os equipamentos utilizados foram cronômetro digital, caneta, prancheta e papel A4 para anotação dos dados. 
Os dados foram inseridos em planilhas do Software Excel versão 2007, e obteve o resultado contabilizado de 85 pacientes em todo o período de pesquisa e aproximadamente 44horas de tempo cronometrado.

\subsection{Procedimentos estatísticos $\mathrm{x}^{-}$}

A análise dos dados obtidos durante o período de coleta utilizou termos como médias, desvio padrão e variação. O fator estatístico $\mathrm{x}^{-}$é a média das médias de cada dia de dados coletados. Para cada dia foi feita uma média dos tempos de atendimentos e depois uma média dessas médias $\left(\mathrm{x}^{-}\right)$.

Para aceitação das médias obtidas pelos dados foram utilizados coeficientes de variação para estipular limites aceitáveis para os dados.

\subsection{A Empresa objeto de Estudo}

O laboratório Labclin Ltda é uma empresa localizada em Cataguases, que iniciou suas atividades em 1996 dentro do Hospital de Cataguases, sendo que este possuía um laboratório próprio para oferecer aos pacientes maiores facilidade de acesso a serviços laboratoriais de análises clínicas.

Passando de próprio para terceirizado por uma funcionária do hospital, a atual sócia administradora do Labclin, recebeu a proposta de abrir um laboratório de análises clínicas para atender ao Hospital e aos pacientes de Cataguases/ MG e região. Nesse momento constituiu uma sociedade por quotas de participação Ltda juntamente com um colega de profissão com o qual exerce, em conjunto, a administração do laboratório.

O Labclin atende aproximadamente 200 pessoas por dia, entre pacientes internos, externos, hemodiálise e pronto - socorro. Sendo realizado cerca de 1.300 exames por dia, com profissionais qualificados, entre técnicos, biomédicos, secretárias, faxineiras e dois sócios proprietários. O laboratório possui 5 balcões de atendimento, uma sala de espera que acomodam cerca de 40 pessoas, sala de café, cabine de coletas, recepção, sala de reuniões e 5 salas de exames.

\section{GERENCIAMENTO DE FILAS}

Segundo Bob Bates, James Mcgrath (2017) ao fazer comparações externas, não é necessário que a organização compare a si mesma com outras organizações do mesmo setor. Por exemplo, os bancos podem aprender muito sobre o gerenciamento de filas, comparando suas práticas com supermercados e estabelecimentos de fastfood. Segundo Krajewski (2012) a fila ocorre porque tem um desnível passageiro entre o nível de demanda de um sistema e a capacidade do sistema em satisfazer esse pedido.

Para Taha (2010) os elementos mais importantes num sistema de filas são os clientes e os atendentes. Chegando ao local, o cliente pode ser atendido na hora ou esperar nas filas, se os atendentes estiverem todos ocupados. Quando um serviço é finalizado, automaticamente a próxima pessoa que estiver na fila é atendida.

\subsection{Sistemas de filas}

Para Mendonça (2014) um sistema com fila é formado de usuários, de canais ou posto de serviço/atendimento e de um espaço designado para a espera. Os usuários chegam num tempo, definido como o processo de chegada, para serem atendidos em um determinado posto de atendimento, enquanto os postos estão com clientes os usuários devem aguardar em uma fila para serem atendidos. Assim que um canal de serviço fica disponível, o próximo usuário que está aguardando na fila é atendido, uma vez terminado o serviço, o usuário já está automaticamente liberado. 


\subsection{Problemas de filas de espera}

Segundo Bitran, Ferrer e Rocha (2008), as filas são um aspecto essencial na gestão de serviços. Filas excessivas são um fator que descontenta clientes e consumidores em todo e qualquer estabelecimento, além disso, gera muitas reclamações por parte destes. Classifica ficando-os em:

- Processo de chegada dos usuários: que normalmente sucede de forma variável e incerta (tempo e quantidade);

- Disciplina da fila: que determina a política de atendimento. Normalmente adota-se a regra First In First Out (FIFO) onde o primeiro a chegar deverá ser o primeiro a ser atendido;

\subsection{Processos de chegada e tempo de atendimento}

O método de chegada indica qual será o parâmetro de chegada dos clientes no sistema de filas. Apresenta comportamento aleatório, ou seja, as chegadas decorrem no tempo e no espaço conforme as leis da probabilidade; desta forma, é preciso conhecer qual distribuição de probabilidade que representa os tempos e as chegadas dos clientes. (AQUILANO, CHASE, JACOBS, 2006).

As equações são classificadas baseando-se nas seguintes características dos processos de chegada e dos atendimentos aos clientes: As chegadas se realizam segundo uma ordenação de Poisson com média $\lambda$ chegadas/tempo. Os tempos de atendimento dispõem a distribuição exponencial de Poisson com média $\mu$. $\mathrm{O}$ número de clientes possíveis é suficientemente grande que a população seja considerada infinita (ARENALES, 2007).

O levantamento de dados estatísticos tem a finalidade definir a distribuição de probabilidades do número de atendimentos ou a duração de cada atendimento.

\section{RESULTADOS}

Foram utilizadas como procedimento as planilhas no software Excel para determinação de médias, desvio padrão, variância e coeficiente de variação. Segundo Montgomery (2009), às vezes é desejável estimar diretamente o desvio padrão em vez do uso da amplitude R. O n (número de amostras) for moderadamente grande, maior que 10 ou 12, utilizou-se os gráficos $\mathrm{x}^{-} \mathrm{e} \mathrm{S}$, onde $\mathrm{S}$ é o desvio padrão amostral.

O Quadro 1 apresenta uma descrição estatística para a construção dos Limites de controle $\mathrm{x}^{-} \mathrm{e} \mathrm{S}$ com um número de amostra variável, onde $\mathrm{S}$ é o desvio padrão amostral e $\mathrm{x}^{-}$ é a média amostral.

Quadro 1- Desvio Padrão S/
\begin{tabular}{|l|l|l|}
\hline Dias & Desvio padrão S & Tempos médios \\
\hline $19 / 11 / 2018$ & 0,003408837 & $00: 07: 18$ \\
\hline $20 / 11 / 2018$ & 0,004402536 & $00: 09: 14$ \\
\hline $21 / 11 / 2018$ & 0,010035051 & $00: 08: 00$ \\
\hline $22 / 11 / 2018$ & 0,00447206 & $00: 08: 42$ \\
\hline $23 / 11 / 2018$ & 0,004869165 & $00: 10: 19$ \\
\hline
\end{tabular}

Fonte: Autores

Os dados apresentados no Quadro-1 obtiveram algumas discrepâncias, nos dias 20 e 23 ficaram acima dos limites estimados. Outra observação importante é que os dados coletados possuem tempos com grandes divergências entre si, isso se deve ao trabalho que é realizado no Laboratório, há exames que demandam mais tempo de atendimento que outros, criando essas diferenças, o fato é que isso é normal no dia a dia de trabalho, por esta razão esses dados não foram eliminados. 
Quadro 2 - Apresentação do sistema FIFO no dia 19/11/2018

\begin{tabular}{|c|c|c|c|c|c|}
\hline \multicolumn{6}{|c|}{ Laboratório Labclin - 19/11/2018 } \\
\hline Paciente & Convênio & $\begin{array}{l}\text { Hora de } \\
\text { Chegada }\end{array}$ & $\begin{array}{l}\text { Início do } \\
\text { Atendimento }\end{array}$ & $\begin{array}{l}\text { Hora de } \\
\text { Saída }\end{array}$ & $\begin{array}{l}\text { Tempo } \\
\text { de } \\
\text { Espera }\end{array}$ \\
\hline PACIENTE 1 & SUS & $06: 40: 00$ & 07:00:00 & 07:05:00 & 00:05:00 \\
\hline PACIENTE 2 & UNIMED & 06:40:00 & 07:06:00 & 07:15:00 & 00:09:00 \\
\hline PACIENTE 3 & SUS & 06:40:00 & 07:17:00 & 07:21:00 & 00:04:00 \\
\hline PACIENTE 4 & SUS & 06:42:00 & 07:23:00 & 07:27:00 & 00:04:00 \\
\hline PACIENTE 5 & SUS & 06:45:00 & 07:30:00 & 07:35:00 & 00:05:00 \\
\hline PACIENTE 6 & SUS & 06:46:00 & 07:36:00 & 07:40:00 & 00:04:00 \\
\hline PACIENTE 7 & PROMED & $06: 50: 00$ & $07: 42: 00$ & 07:59:00 & 00:17:00 \\
\hline PACIENTE 8 & SUS & 06:53:00 & 08:02:00 & 08:06:00 & 00:04:00 \\
\hline PACIENTE 9 & VITALLIS & 06:58:00 & 08:07:00 & 08:16:00 & 00:09:00 \\
\hline PACIENTE 10 & PARTICULAR & 07:06:00 & 08:17:00 & 08:22:00 & 00:05:00 \\
\hline PACIENTE 11 & UNIMED & 07:10:00 & 08:24:00 & 08:29:00 & 00:05:00 \\
\hline PACIENTE 12 & PROMED & 07:16:00 & 08:31:00 & 08:38:00 & 00:07:00 \\
\hline PACIENTE 13 & SUS & 07:23:00 & 08:40:00 & 08:44:00 & 00:04:00 \\
\hline PACIENTE 14 & SUS & 07:27:00 & 08:45:00 & 08:49:00 & 00:04:00 \\
\hline PACIENTE 15 & SUS & 07:34:00 & 08:51:00 & 08:55:00 & 00:04:00 \\
\hline PACIENTE 16 & CASSI & 07:40:00 & 08:56:00 & 09:13:00 & 00:17:00 \\
\hline PACIENTE 17 & CASSI & 07:46:00 & 09:14:00 & 09:31:00 & $00: 17: 00$ \\
\hline \multicolumn{5}{|l|}{ MEDIA } & 00:07:18 \\
\hline
\end{tabular}

Fonte: Autores

De acordo com o Quadro 2, está sendo analisado atendimentos no método FIFO (First in - First out). Com esse método, pode-se observar que o paciente de número 1 , portador do convênio SUS, chegou às 06:40hs, sendo atendido às 07:00hs, com o término de seu atendimento às 07:05hs. Seu tempo de espera foi de 5 minutos. Dessa forma foi demostrado sucessivamente os demais atendimentos, independente do seu convênio, com um tempo médio total de espera de 7 minutos e 18 segundos. 
Quadro 3 - Apresentação do sistema FIFO no dia 20/11/2018

\begin{tabular}{|c|c|c|c|c|c|}
\hline \multicolumn{6}{|c|}{ Laboratório Labclin - 20/11/2018 } \\
\hline Paciente & Convênio & $\begin{array}{l}\text { Hora de } \\
\text { Chegada }\end{array}$ & $\begin{array}{l}\text { Início do } \\
\text { Atendimento }\end{array}$ & \begin{tabular}{|l|} 
Hora \\
Saída
\end{tabular} & $\begin{array}{l}\text { Tempo de } \\
\text { Espera }\end{array}$ \\
\hline $\begin{array}{l}\text { PACIENTE } \\
18\end{array}$ & UNIMED & 06:55:00 & 07:00:00 & 07:11:00 & 00:11:00 \\
\hline $\begin{array}{l}\text { PACIENTE } \\
19\end{array}$ & UNIMED & 06:55:00 & 07:13:00 & $07: 21: 00$ & 00:08:00 \\
\hline $\begin{array}{l}\text { PACIENTE } \\
20\end{array}$ & SUS & 06:57:00 & 07:22:00 & $07: 26: 00$ & 00:04:00 \\
\hline $\begin{array}{l}\text { PACIENTE } \\
21\end{array}$ & SUS & 06:58:00 & 07:27:00 & $07: 31: 00$ & 00:04:00 \\
\hline $\begin{array}{l}\text { PACIENTE } \\
22\end{array}$ & SUS & 07:00:00 & 07:32:00 & $07: 35: 00$ & 00:03:00 \\
\hline $\begin{array}{l}\text { PACIENTE } \\
23\end{array}$ & PROMED & 07:01:00 & 07:36:00 & 07:48:00 & 00:12:00 \\
\hline $\begin{array}{l}\text { PACIENTE } \\
24\end{array}$ & AMIL & 07:01:00 & 07:50:00 & 08:09:00 & 00:19:00 \\
\hline $\begin{array}{l}\text { PACIENTE } \\
25\end{array}$ & SUS & 07:03:00 & 08:10:00 & $08: 15: 00$ & 00:05:00 \\
\hline $\begin{array}{l}\text { PACIENTE } \\
26\end{array}$ & SUS & 07:07:00 & 08:16:00 & 08:19:00 & 00:03:00 \\
\hline $\begin{array}{l}\text { PACIENTE } \\
27\end{array}$ & PARTICULAR & 07:10:00 & 08:20:00 & 08:29:00 & 00:09:00 \\
\hline $\begin{array}{l}\text { PACIENTE } \\
28\end{array}$ & S. AMERICA & 07:25:00 & 08:30:00 & $08: 45: 00$ & 00:15:00 \\
\hline $\begin{array}{l}\text { PACIENTE } \\
29\end{array}$ & PROMED & 07:28:00 & 08:43:00 & 08:53:00 & 00:10:00 \\
\hline $\begin{array}{l}\text { PACIENTE } \\
30\end{array}$ & SUS & $07: 35: 00$ & 08:54:00 & 08:59:00 & 00:05:00 \\
\hline $\begin{array}{l}\text { PACIENTE } \\
31\end{array}$ & SUS & $07: 41: 00$ & 09:00:00 & 09:06:00 & 00:06:00 \\
\hline $\begin{array}{l}\text { PACIENTE } \\
32\end{array}$ & SUS & 08:00:00 & 09:02:00 & 09:06:00 & 00:04:00 \\
\hline $\begin{array}{l}\text { PACIENTE } \\
33\end{array}$ & POLICIA & 08:05:00 & 09:09:00 & 09:35:00 & 00:26:00 \\
\hline $\begin{array}{l}\text { PACIENTE } \\
34\end{array}$ & CASSI & 08:12:00 & 09:36:00 & 09:49:00 & 00:13:00 \\
\hline MÉDIA & & & & & $00: 09: 14$ \\
\hline
\end{tabular}

Fonte: Autores

De acordo com o Quadro 3, está sendo analisado atendimentos no método FIFO (First in - First out). Com esse método, pode-se observar que o paciente de número 18, portador do convênio Unimed, chegou às $06: 55 \mathrm{hs}$, sendo atendido às $07: 00 \mathrm{hs}$, com o término de seu atendimento às 07:11hs. Seu tempo de espera foi de 11 minutos. Dessa forma foi demostrado sucessivamente os demais atendimentos, independente do seu convênio, com um tempo total médio de espera de 9 minutos e 14 segundos. 
Quadro 4 - Apresentação do sistema FIFO no dia 21/11/2018

\begin{tabular}{|c|c|c|c|c|c|}
\hline \multicolumn{6}{|c|}{ Laboratório Labclin - 21/11/2018 } \\
\hline Paciente & Convênio & $\begin{array}{l}\text { Hora de } \\
\text { Chegada }\end{array}$ & $\begin{array}{l}\text { Início do } \\
\text { Atendimento }\end{array}$ & $\begin{array}{l}\text { Hora de } \\
\text { Saída }\end{array}$ & $\begin{array}{l}\text { Tempo de } \\
\text { Espera }\end{array}$ \\
\hline ACIENTE 35 & SUS & 06:45:00 & 07:00:00 & 07:05:00 & 00:05:00 \\
\hline $\begin{array}{l}\text { PACIENTE } \\
36\end{array}$ & SUS & 06:45:00 & 07:06:00 & 07:10:00 & 00:04:00 \\
\hline $\begin{array}{l}\text { PACIENTE } \\
37\end{array}$ & SUS & 06:46:00 & 07:11:00 & $07: 16: 00$ & 00:05:00 \\
\hline $\begin{array}{l}\text { PACIENTE } \\
38 \\
\end{array}$ & UNIMED & 06:54:00 & 07:18:00 & 07:28:00 & 00:10:00 \\
\hline $\begin{array}{l}\text { PACIENTE } \\
39\end{array}$ & SUS & 07:05:00 & 07:30:00 & 07:35:00 & 00:05:00 \\
\hline $\begin{array}{l}\text { PACIENTE } \\
40\end{array}$ & AMIL & 07:05:00 & 07:36:00 & 07:49:00 & 00:13:00 \\
\hline $\begin{array}{l}\text { PACIENTE } \\
41\end{array}$ & SUS & 07:09:00 & 07:50:00 & 07:55:00 & 00:05:00 \\
\hline $\begin{array}{l}\text { PACIENTE } \\
42\end{array}$ & SUS & 07:13:00 & 07:57:00 & 08:01:00 & 00:04:00 \\
\hline $\begin{array}{l}\text { PACIENTE } \\
43\end{array}$ & SUS & 07:20:00 & 08:03:00 & 08:07:00 & 00:04:00 \\
\hline $\begin{array}{l}\text { PACIENTE } \\
44\end{array}$ & PARTICULAR & 07:26:00 & 08:08:00 & 08:14:00 & 00:06:00 \\
\hline $\begin{array}{l}\text { PACIENTE } \\
45\end{array}$ & UNIMED & 07:35:00 & 08:16:00 & 08:28:00 & 00:12:00 \\
\hline $\begin{array}{l}\text { PACIENTE } \\
46\end{array}$ & PROMED & 07:45:00 & 08:30:00 & 08:45:00 & $00: 15: 00$ \\
\hline $\begin{array}{l}\text { PACIENTE } \\
47\end{array}$ & SUS & 08:00:00 & 08:47:00 & 08:51:00 & 00:04:00 \\
\hline $\begin{array}{l}\text { PACIENTE } \\
48\end{array}$ & SUS & 08:11:00 & 08:53:00 & 08:59:00 & 00:06:00 \\
\hline $\begin{array}{l}\text { PACIENTE } \\
49\end{array}$ & SUS & 08:19:00 & 09:02:00 & 09:06:00 & 00:04:00 \\
\hline $\begin{array}{l}\text { PACIENTE } \\
50\end{array}$ & CASSI & 08:30:00 & 09:11:00 & 09:31:00 & 00:20:00 \\
\hline $\begin{array}{l}\text { PACIENTE } \\
51\end{array}$ & VIATALLIS & 08:34:00 & 09:33:00 & 09:50:00 & $00: 17: 00$ \\
\hline $\begin{array}{l}\text { PACIENTE } \\
52\end{array}$ & SUS & 08:34:00 & 09:53:00 & 09:58:00 & 00:05:00 \\
\hline MÉDIA & & & & & 00:08:00 \\
\hline
\end{tabular}

Fonte: Autores

De acordo com o Quadro 4, está sendo analisado atendimentos no método FIFO (First in - First out). Com esse método, pode-se observar que o paciente de número 35, portador do convênio SUS, chegou às $06: 45 \mathrm{hs}$, sendo atendido às 07:00hs, com o término de seu atendimento às 07:05hs. Seu tempo de espera foi de 5 minutos. Dessa forma foi demostrado sucessivamente os demais atendimentos, independente do seu convênio, com um tempo médio total de espera de 8 minutos. 
Quadro 5 - Apresentação do sistema FIFO no dia 22/11/2018

\begin{tabular}{|l|l|l|l|l|l|}
\hline Laboratório Labclin - 22/11/2018 \\
\hline Paciente & Convênio & $\begin{array}{l}\text { Hora de } \\
\text { Chegada }\end{array}$ & $\begin{array}{l}\text { Início do do } \\
\text { Atendimento }\end{array}$ & $\begin{array}{l}\text { Hora } \\
\text { Saída }\end{array}$ & $\begin{array}{l}\text { Tempo de } \\
\text { Espera }\end{array}$ \\
\hline PACIENTE 53 & SUS & $06: 40: 00$ & $06: 59: 00$ & $07: 04: 00$ & $00: 05: 00$ \\
\hline PACIENTE 54 & SUS & $06: 40: 00$ & $07: 05: 00$ & $07: 09: 00$ & $00: 04: 00$ \\
\hline PACIENTE 55 & SUS & $06: 41: 00$ & $07: 10: 00$ & $07: 15: 00$ & $00: 05: 00$ \\
\hline PACIENTE 56 & SUS & $06: 42: 00$ & $07: 16: 00$ & $07: 20: 00$ & $00: 04: 00$ \\
\hline PACIENTE 57 & SUS & $06: 42: 00$ & $07: 21: 00$ & $07: 26: 00$ & $00: 05: 00$ \\
\hline PACIENTE 58 & CASSI & $06: 55: 00$ & $07: 28: 00$ & $07: 46: 00$ & $00: 18: 00$ \\
\hline PACIENTE 59 & SUS & $06: 59: 00$ & $07: 48: 00$ & $07: 54: 00$ & $00: 06: 00$ \\
\hline PACIENTE 60 & SUS & $07: 03: 00$ & $07: 55: 00$ & $08: 00: 00$ & $00: 05: 00$ \\
\hline PACIENTE 61 & SUS & $07: 10: 00$ & $08: 02: 00$ & $08: 06: 00$ & $00: 04: 00$ \\
\hline PACIENTE 62 & PROMED & $07: 21: 00$ & $08: 08: 00$ & $08: 30: 00$ & $00: 22: 00$ \\
\hline PACIENTE 63 & UNIMED & $07: 28: 00$ & $08: 32: 00$ & $08: 45: 00$ & $00: 13: 00$ \\
\hline PACIENTE 64 & PROMED & $07: 29: 00$ & $08: 47: 00$ & $09: 09: 00$ & $00: 22: 00$ \\
\hline PACIENTE 65 & SUS & $07: 32: 00$ & $09: 10: 00$ & $09: 15: 00$ & $00: 05: 00$ \\
\hline PACIENTE 66 & SUS & $07: 49: 00$ & $09: 17: 00$ & $09: 23: 00$ & $00: 06: 00$ \\
\hline PACIENTE 67 & SUS & $08: 00: 00$ & $09: 25: 00$ & $09: 30: 00$ & $00: 05: 00$ \\
\hline PACIENTE 68 & CASSI & $08: 15: 00$ & $09: 32: 00$ & $09: 46: 00$ & $00: 14: 00$ \\
\hline PACIENTE 69 & SUS & $08: 23: 00$ & $09: 50: 00$ & $09: 55: 00$ & $00: 05: 00$ \\
\hline MÉDIA & & & & $00: 08: 42$ \\
\hline
\end{tabular}

Fonte: Autores

De acordo com o Quadro 5, está sendo analisado atendimentos no método FIFO (First in - First out). Com esse método, pode-se observar que o paciente de número 53, portador do convênio SUS, chegou às $06: 40 \mathrm{hs}$, sendo atendido às 06:59hs, com o término de seu atendimento às 07:04hs. Seu tempo de espera foi de 5 minutos. Dessa forma foi demostrado sucessivamente os demais atendimentos, independente do seu convênio, com um tempo médio total de espera de 8 minutos e 42 segundos. 
Quadro 6 - Apresentação do sistema FIFO no dia 23/11/2018

\begin{tabular}{|l|l|l|l|l|l|}
\hline Laboratório Labclin - 23/11/2018 & $\begin{array}{l}\text { Tempo de } \\
\text { Paciente }\end{array}$ & Convênio & $\begin{array}{l}\text { Início do } \\
\text { Atendimento }\end{array}$ & $\begin{array}{l}\text { Hora } \\
\text { Saída }\end{array}$ & $\begin{array}{l}\text { de } \\
\text { Espera }\end{array}$ \\
\hline $\begin{array}{l}\text { PACIENTE } \\
70\end{array}$ & AMIL & $06: 50: 00$ & $07: 00: 00$ & $07: 25: 00$ & $00: 25: 00$ \\
\hline $\begin{array}{l}\text { PACIENTE } \\
71\end{array}$ & UNIMED & $06: 51: 00$ & $07: 26: 00$ & $07: 38: 00$ & $00: 12: 00$ \\
\hline $\begin{array}{l}\text { PACIENTE } \\
72\end{array}$ & SUS & $06: 55: 00$ & $07: 40: 00$ & $07: 45: 00$ & $00: 05: 00$ \\
\hline $\begin{array}{l}\text { PACIENTE } \\
73\end{array}$ & SUS & $07: 00: 00$ & $07: 47: 00$ & $07: 52: 00$ & $00: 05: 00$ \\
\hline $\begin{array}{l}\text { PACIENTE } \\
74\end{array}$ & SUS & $07: 06: 00$ & $07: 54: 00$ & $07: 59: 00$ & $00: 05: 00$ \\
\hline $\begin{array}{l}\text { PACIENTE } \\
75\end{array}$ & SUS & $07: 15: 00$ & $08: 01: 00$ & $08: 06: 00$ & $00: 05: 00$ \\
\hline $\begin{array}{l}\text { PACIENTE } \\
76\end{array}$ & POLICIA & $07: 26: 00$ & $08: 08: 00$ & $08: 29: 00$ & $00: 21: 00$ \\
\hline $\begin{array}{l}\text { PACIENTE } \\
77\end{array}$ & SUS & $07: 33: 00$ & $08: 33: 00$ & $08: 39: 00$ & $00: 06: 00$ \\
\hline $\begin{array}{l}\text { PACIENTE } \\
78\end{array}$ & IPSEMG & $07: 40: 00$ & $08: 41: 00$ & $08: 59: 00$ & $00: 18: 00$ \\
\hline $\begin{array}{l}\text { PACIENTE } \\
79\end{array}$ & PARTICULAR & $07: 43: 00$ & $09: 02: 00$ & $09: 10: 00$ & $00: 08: 00$ \\
\hline $\begin{array}{l}\text { PACIENTE } \\
80\end{array}$ & UNIMED & $08: 00: 00$ & $09: 13: 00$ & $09: 20: 00$ & $00: 07: 00$ \\
\hline $\begin{array}{l}\text { PACIENTE } \\
81\end{array}$ & PROMED & $08: 15: 00$ & $09: 22: 00$ & $09: 37: 00$ & $00: 15: 00$ \\
\hline $\begin{array}{l}\text { PACIENTE } \\
82\end{array}$ & SUS & $08: 26: 00$ & $09: 40: 00$ & $09: 46: 00$ & $00: 06: 00$ \\
\hline $\begin{array}{l}\text { PACIENTE } \\
83\end{array}$ & SUS & $08: 34: 00$ & $09: 50: 00$ & $09: 55: 00$ & $00: 05: 00$ \\
\hline $\begin{array}{l}\text { PACIENTE } \\
84\end{array}$ & SUS & $08: 42: 00$ & $09: 57: 00$ & $10: 00: 00$ & $00: 03: 00$ \\
\hline $\begin{array}{l}\text { PACIENTE } \\
85\end{array}$ & CASSI & $09: 00: 00$ & $10: 03: 00$ & $10: 22: 00$ & $00: 19: 00$ \\
\hline MÉDIA & & & & $00: 10: 19$ \\
\hline
\end{tabular}

Fonte: Autores

De acordo com o Quadro 6, está sendo analisado atendimentos no método FIFO (First in - First out). Com esse método, pode-se observar que o paciente de número 70, portador do convênio AMIL, chegou às 06:50hs, sendo atendido às 07:00hs, com o término de seu atendimento às 07:25hs. Seu tempo de espera foi de 25 minutos. Dessa forma foi demostrado sucessivamente os demais atendimentos, independente do seu convênio, com um tempo médio total de espera de 10 minutos e 19 segundos.

Os cálculos dos parâmetros dos Limites $x^{-}$e $S$, onde a média dos subgrupos (n/) igual a 5. O Quadro 6 representa os cálculos dos Limites de controle S, onde S/ é igual a 0, 00544. 
Quadro 7- Cálculos dos Limites S

$$
\begin{array}{lll}
\text { Limite superior de controle } & =B_{4} \times \mathrm{S} / & 0,01157 \\
\text { Limite central de controle } & =\mathrm{S} / & 0,00544 \\
\text { Limite inferior de controle } & =B_{3} \times \mathrm{S} / & 0,00069
\end{array}
$$

Onde: $B 4=1+3 / C 4 \sqrt{2}$.(n-1), B3 = $1-3 / C 4 \sqrt{2}(n-1)$ e $C 4=4(n-1) / 4 n-3$.

Os parâmetros para os limites do desvio padrão (Quadro 6) são $B_{4} \mathrm{e} B_{3}$,obtidos através do cálculo acima, os limites encontrados se fazem necessários para interpretação do Quadro 1.

Após cronometrar o tempo de chegada, atendimento e saída dos pacientes, foi implantado o método SJF-NP durante 3 horas de atendimento, fazendo com que o tempo de atendimento reduzisse em cerca de 39 minutos, com isso pode-se observar a utilidade da ferramenta no Laboratório Labclin.

Quadro 8 - Apresentação do sistema SJF-NP no dia 23/11/2018

\begin{tabular}{|l|l|l|l|l|l|}
\hline Laboratório Labclin - 23/11/2018 \\
\hline Paciente & Convênio & $\begin{array}{l}\text { Hora de } \\
\text { Chegada }\end{array}$ & $\begin{array}{l}\text { Início do do } \\
\text { Atendimento }\end{array}$ & $\begin{array}{l}\text { Hora } \\
\text { Saída }\end{array}$ & $\begin{array}{l}\text { Tempo } \\
\text { Espera }\end{array}$ \\
\hline PACIENTE 72 & SUS & $06: 55: 00$ & $07: 00: 00$ & $07: 05: 00$ & $00: 05: 00$ \\
\hline PACIENTE 70 & AMIL & $06: 50: 00$ & $07: 05: 00$ & $07: 30: 00$ & $00: 25: 00$ \\
\hline PACIENTE 73 & SUS & $07: 00: 00$ & $07: 30: 00$ & $07: 35: 00$ & $00: 05: 00$ \\
\hline PACIENTE 74 & SUS & $07: 06: 00$ & $07: 35: 00$ & $07: 40: 00$ & $00: 05: 00$ \\
\hline PACIENTE 75 & SUS & $07: 15: 00$ & $07: 40: 00$ & $07: 45: 00$ & $00: 05: 00$ \\
\hline PACIENTE 77 & SUS & $07: 33: 00$ & $07: 45: 00$ & $07: 51: 00$ & $00: 06: 00$ \\
\hline PACIENTE 71 & UNIMED & $06: 51: 00$ & $07: 51: 00$ & $08: 03: 00$ & $00: 12: 00$ \\
\hline PACIENTE 76 & POLICIA & $07: 26: 00$ & $08: 03: 00$ & $08: 24: 00$ & $00: 21: 00$ \\
\hline PACIENTE 78 & IPSEMG & $07: 40: 00$ & $08: 24: 00$ & $08: 42: 00$ & $00: 18: 00$ \\
\hline PACIENTE 82 & SUS & $08: 26: 00$ & $08: 42: 00$ & $08: 48: 00$ & $00: 06: 00$ \\
\hline PACIENTE 83 & SUS & $08: 34: 00$ & $08: 48: 00$ & $08: 53: 00$ & $00: 05: 00$ \\
\hline PACIENTE 84 & SUS & $08: 42: 00$ & $08: 53: 00$ & $08: 56: 00$ & $00: 03: 00$ \\
\hline PACIENTE 79 & PARTICULAR & $07: 43: 00$ & $08: 56: 00$ & $09: 04: 00$ & $00: 08: 00$ \\
\hline PACIENTE 80 & UNIMED & $08: 00: 00$ & $09: 04: 00$ & $09: 11: 00$ & $00: 07: 00$ \\
\hline PACIENTE 81 & PROMED & $08: 15: 00$ & $09: 11: 00$ & $09: 26: 00$ & $00: 15: 00$ \\
\hline PACIENTE 85 & CASSI & $09: 00: 00$ & $09: 26: 00$ & $09: 45: 00$ & $00: 19: 00$ \\
\hline MÉDIA & & & & $00: 09: 40$ \\
\hline
\end{tabular}

Fonte: Autores

De acordo com o Quadro 8, está sendo analisado atendimentos no método SJF-NP (Shortest Job First - Não preemptivo). Com esse método, pode-se observar que os pacientes portadores do convênio SUS, tiveram um atendimento mais rápido, diminuindo o tempo de espera dos demais na fila. Dessa forma foi demostrado sucessivamente os outros atendimentos, independente do seu convênio, com um tempo médio total de espera de 9 minutos e 40 segundos.

Quadro 9 - Cálculos dos Limites $X^{-}$

Limite superior de controle

Limite central de controle

$$
=X^{+x A_{3} x S}
$$

Limite inferior de controle
$=X^{-}$

$$
=X^{-x A_{3} x S}
$$

$8,723 \mathrm{~min}$

$8,716 \mathrm{~min}$

$8,720 \mathrm{~min}$

\section{8:43:23}

8:43:12 
Onde: $\mathrm{A} 3=3 / \mathrm{C} 4 \sqrt{ } \mathrm{n}$.

O Quadro 9 possui os limites de $X^{-}$, assim como o desvio padrão das médias devem estar dentro de um limite aceitável para aceitação dos dados (Quadro 1).

Os atendimentos feitos para o SUS (Sistema Único de Saúde), são pré-aprovados e por esse motivo os atendimentos realizados com maior rapidez. Atendimentos que possuem convênio precisam que a atendente cadastre o pedido em um servidor online do respectivo convênio, que por inúmeras razões fica fora de serviço.

A solução possível seria utilizar uma das ferramentas da Pesquisa Operacional com um modelo de gerenciamento de filas SJF - NP. O laboratório poderá dispor de um balcão para atendimento com o funcionamento do sistema SJF - NP, onde os pacientes do SUS terão prioridade, uma vez que são processados mais rápidos. Para evitar conflitos de pacientes, no momento que não tiver nenhum espera do SUS, poderá ser dado andamento aos demais atendimentos.

Quadro 10 - Demonstrativo da média dos dias analisados, com métodos FIFO aplicados todos os dias, e o SJF-NP apenas dia 23/11/2018.

\begin{tabular}{|l|l|l|l|l|l|}
\hline Data & 19 & 20 & 21 & 22 & 23 \\
\hline Método & $00: 07: 18$ & $00: 09: 14$ & $00: 08: 00$ & $00: 08: 42$ & $00: 10: 19$ \\
\hline FIFO & & & & & $00: 09: 40$ \\
\hline SJF-NP
\end{tabular}

Fonte: Autores

\section{5- CONSIDERAÇÕES FINAIS}

Com os resultados obtidos na pesquisa do sistema de gerenciamento de filas que ocorre no atendimento do laboratório Labclin, observou-se que apesar do processo de chegada dos usuários acontecerem normalmente de forma variável e incerta, pacientes que são destinados a atendimentos específicos como SUS, convênios ou particular, o tempo de espera varia, de acordo com o exame que o paciente fará, sendo o atendimento pelo SUS a maior demanda dos dias de trabalho analisados (19/11 a 23/11).

Observa-se que, a maior procura pelos serviços dá-se pelos clientes do SUS, onde verificou-se a necessidade de um atendimento exclusivo para esse público, que diminuiria as filas e aperfeiçoaria o atendimento destas pessoas, uma vez que esse atendimento é mais rápido e possui maior demanda, com isso torna-se viável para o laboratório adotar um sistema de Gerenciamento de Filas.

A Pesquisa Operacional tem como objetivo principal utilizar uma de suas ferramentas da teoria do Gerenciamento de Filas para solucionar os problemas em relação aos pacientes com limites acima do tempo de espera nas filas.

Em relação ao tempo médio dos atendimentos, pôde-se observar que os dados apresentaram algumas discrepâncias conforme observado no Quadro1. Nos dias 20 e 23 os dados ficaram acima dos limites estimados, limites estes estipulados por cálculos com limites superiores de controle de 8,723 minutos e limite inferior de 8,720 minutos.

\section{REFERÊNCIAS BIBLIOGRÁFICAS}

AQUILANO, N.J.; CHASE, R.B.; JACOBS, F.R. Administração da Produção e Operações para Vantagens Competitivas. 11 $1^{\text {a }}$.ed. São Paulo, 2006.

ARENALES, Armentano, Morabito, Yanasse. Pesquisa Operacional. 6 ${ }^{a}$ Tiragem, ED; Campus- Abrepro, Rio de Janeiro, RJ. 2007.

BITRAN, Gabriel R.; FERRER, Juan-Carlos; ROCHA E OLIVEIRA, Paulo. OM forum- managing customer experiences: Perspectives on the temporal aspects of service encounters. Manufacturing \& Service Operations Management, v. 10, n. 1, p. 61-83, 2008. 
BOB BATES, James Mcgrath -Gestão da Produção e Logística - SÉRIE GESTÃO EMPRESARIAL. 2017

RITZMAN, L. P. \& KRAJEWSKI, L. J. Administração da produção e operações, $8^{a}$. ed. São Paulo: Pearson Prentice Hall, 2012.

MENDONÇA, E. Teoria de filas markovianas e aplicações, 2014. Disponível em:

http://dspace.bc.uepb.edu.br/jspui/bitstream/123456789/5265/1/PDF\%20-

\%20Edn\%C3\%A1rio\%20Barbosa\%20de\%20Mendon\%C3\%A7a.pdf. Acesso em março 2019.

MONTGOMERY, Douglas C. Introdução ao Controle Estatístico da Qualidade. 4. ed. Rio de Janeiro: LTC, 2009. $513 \mathrm{p}$.

Roberto José Bittencourt, médico e doutorando da ENSP. Em artigo na revista Cadernos de Saúde Pública (Vol. 25. $\mathrm{N}^{\circ} 7$ )"

TAHA, H. A. Operations Research: An Introduction, 9a ed. Pearson Prentice Hall, 2010. 